\title{
Laparoscopic Intervention for Cases of Adhesive Small Bowel Obstruction: Is It Safe and Beneficial?
}

\author{
Wael E. Lotfy, MD; Basem Sieda, MD; Abdel Wahab Saleh, MD.
}

\section{General surgery department, Zagazig University Hospitals, Zagazig, Egypt.}

Objective: The objective of the study was to evaluate the safety and benefit of laparoscopic management of adhesive small bowel obstruction in order to establish an optimal operative strategy for those patients.

Methods: Between April 2012 to September 2014, our study included 56 patients who presented with acute adhesive small bowel obstruction required surgical intervention. We performed laparoscopy for all patients and performed laparoscopic adhesiolysis whenever possible (LAP Group). Otherwise we converted to open surgery (CONV Group) and we compared the outcome of the two groups and evaluated the risk and benefit of initial laparoscopy.

Results: 56 patients were enrolled in this study. Laparoscopic techniques (LAP) alone were successfully used to complete 40 cases (73\%). Sixteen patients (28\%) were converted to open surgery through midline laparotomies. A single band was identified in 38 patients. Complications were comparable between the two groups however the laparoscopic cases benefited from shorter operative times, fewer wound complications (infection/hernia), quicker recovery, shorter hospital stay, and less pain.

Conclusion: Laparoscopy has a valuable role for cases presented with adhesive small bowel obstruction, specifically for those patients with a postoperative single band causing obstruction. So, most patients with adhesive intestinal obstruction are candidates for laparoscopy. A significant number of patients will be spared a large laparotomy incision.

Key words: Adhesive intestinal obstruction, laparoscopy, adhesiolysis.

\section{Introduction:}

Laparoscopic management of adhesive small bowel obstruction has been shown to be feasible and advantageous. ${ }^{1}$ Patients with single previous operation or patients post appendectomy are the most common patients candidates for laparoscopic adhesiolysis in the event of postoperative adhesive small bowel obstruction (SBO). ${ }^{2}$ Conversion to open surgery should not be considered as failure. ${ }^{3}$

No specific preoperative test is available that will clearly identify those patients who will benefit from laparoscopic techniques. ${ }^{4}$ Obviously, those patients with single adhesion band can be spared unnecessary midline incision by identifying them with an initial laparoscopic approach. ${ }^{5}$ Benefits include shorter operative times, less adhesion formation, fewer wound complications (infection/hernia), quicker recovery, shorter hospital stay, and less pain. 6 The purpose of this study was to prospectively evaluate the feasibility and advantage of initial laparoscopic management of adhesive small bowel obstruction in order to establish an optimal operative strategy.

\section{Patients and methods:}

We prospectively followed 56 consecutive cases of adhesive small bowel obstruction admitted to the emergency unit of Zagazig University hospital from April 2012 to September 2014 who were finally required surgical intervention

Inclusion criteria:

1- Patients' age between 18 and 70 years. 
2- Patients presented with symptoms and signs of acute intestinal obstruction with history of pervious laparotomy.

\section{Exclusion criteria:}

1- Patients below 18 or above 70 years old

2-Patients with no history of previous laparotomy

3- Patients who show signs of strangulation

4- Patients who show signs of peritonitis or those who showed free intraperitoneal gas in plain X ray.

5- Patients who responded to conservative measures and relieved without surgical intervention.

6- Patients in whom laparoscopy revealed the cause of obstruction other than adhesions such as malignancy

All patients had a plain erect X-Ray abdomen and a computed tomographic (CT) scan of the abdomen and pelvis in the first 24 hours. Results for all patients were consistent with small bowel mechanical obstruction.

All patients received conservative measures in the form of nasogastric suction with restriction of oral intake, intravenous fluids with correction of blood electrolytes, prophylactic antibiotics together with repeated enemata. Conservative measures were continued for 24 hours. Patients relieved under these measures were excluded from the study i.e. All patients included in this study failed the trial of conservative management in the first 24 hours.

\section{Operative work:}

Our operative technique was similar in all cases .After induction of general endotracheal anesthesia; patients were placed in the supine position with both arms placed by their sides. Tilting of the table allows the use of gravity to assist in manipulation of the bowel. Typically, the heavier, distended bowel will fall away from the laparoscopic field of vision. Monitors were placed on each side of the table to facilitate shifts in the procedure from quadrant to quadrant. Nasogastric and urinary catheters were placed routinely if not already in place. A prophylactic antibiotic was administered within half an hour preoperatively; the abdomen is draped and prepared in ordinary fashion, then Veress needle was typically placed anyway from surgical scars but mostly in the left upper quadrant, this is generally an area free of adhesions except in patients who had prior left upper quadrant surgery. If unsuccessful, a Hasson or open technique was used. Pneumoperitoneum is established and $10 \mathrm{~mm}$ trocar was inserted for the camera. If open access was not possible, the operation was converted to open surgery. A $10-\mathrm{mm}$, 30-degree angled laparoscope was used in all cases. The remaining laparoscopic ports were then inserted under direct vision. When the distal ileum is suspected to be the site of transition, left lateral port was introduced. Whenever possible, trocars were placed away from the site of interest to triangulate with the camera. Typically, three 5-mm trocars were used. An additional fourth disposable 5-mm was inserted when practically indicated to assist with retraction.

An initial assessment is made to determine the likelihood of successful laparoscopic management. The types, shapes and extents of adhesions encountered after initial insertion of the laparoscope were extremely variable Figures $(1,2,3)$.

Adequate visualization is obtained by some degree of adhesiolysis. The next step, when possible was identification of the decompressed bowel. When a single band was identified with a clear transition zone, the band was lysed and the operation was concluded. If an abnormal loop of bowel was identified, this became the focus of attention. Identification of non-viable bowel necessitated conversion to open surgery. When the transitional zone was not clearly identified, the bowel was run using a "grasper over grasper technique", typically from ileocecal junction to proximal jejunum.. Care was taken to gently manipulate the bowel. Grasping the bowel wall itself was avoided as much as possible and grasping only mesentery or simply pushing the bowel was preferred to avoid iatrogenic injury. Serosal tears and minor injuries with only minimal spillage were repaired with laparoscopic suturing, 
cases with large tears and marked spillage, it was unsafe to proceed laparoscopically, and in these cases, we resorted to midline laparotomy.

During the process of thorough inspection and manipulation of the bowel, resistance or difficulty in pulling the bowel was typically encountered when getting close to the point of obstruction. Lysis of adhesions can be safely performed with the use of laparoscopic bipolar diathermy and scissors Figure (4). Thin adhesions were usually avascular and did not necessarily require surgical clips, or stapling devices for transection Figures $(\mathbf{5 , 6})$. Upon relief of the obstruction, the entire small bowel was re-examined for exclusion of intestinal injury or other sites of obstruction. It was not necessary to lyse all adhesions if a transition zone was clearly identified. In our study, we did not consider conversion to open surgery as a failure.

\section{Postoperative care:}

All patients were postoperatively managed in the inpatient ward of the surgical emergency unit. Intravenous fluids, systemic antibiotics and analgesics were given in their usual doses. Vital data, urine output and central venous pressure were regularly measured for critical cases. Nasogastric tube was withdrawn when intestinal sounds were heard and oral feeding was started in cases that didn't have intestinal injuries. Those patients with intestinal injuries were kept NPO for three days then started oral fluids. Patients were considered relieved if they passed flatus and stool after they had started solid diet with disappearance of clinical and radiological signs of obstruction. Then patients were discharged from hospital on scheduled regular follow up visits in the outpatient clinic.

\section{Results:}

Fifty six patients who presented with acute adhesive small bowel obstruction were enrolled in this study. Patients' ages ranged from 21 to 67 years with mean age $46.5 \pm 23.5$ years. There were 27 males and 29 females.

They underwent initial laparoscopy. Out of fifty six patients, 40 patients (73\%) had definitive, completely laparoscopic adhesiolysis (LAP group). Sixteen patients $(27 \%)$ were converted to standard midline laparotomy (CONV group).

All patients had previous abdominal surgery, 39 patients $(70 \%)$ had previous single surgery and 17 patients $(30 \%)$ have more than one surgery.. 34 patients out of 39 patients (87\%) with single prior operation were treated laparoscopically without conversion to laparotomy. And the remaining 5 patients were converted to open surgery. Out of the 17 patients who had more than one surgery, only 6 patients had complete laparoscopic adhesiolysis and 11 patients were converted to open surgery.

The first former surgical operation was appendectomy in 37 patients $(66 \%)$, Hemicolectomy in 6 patients (10.8\%), hysterectomy in 6 patients $(10.8 \%)$ splenectomy in 3 patients (5.3); umbilical hernia in 3 patients $(5.3 \%)$ and inguinal hernia, in 1 patient (1.8\%) Table (1).

The factors predicting the success of laparoscopic approach in management of adhesive SBO are shown in Table (2). It is clear that single laparotomy, early laparoscopy within 48 hours, appendectomy as a primary operation and non-median laparotomy are the favorable predictive factors of successful laparoscopic adhesiolysis.

The adhesion accused of obstruction was classified into isolated band in 38 patients $(67.8 \%)$ and multiple adhesions in 18 patients $(32.2 \%)$.

Causes of conversion to open adhesiolysis in the 16 patients were multiple serosal tears in 7 patients, dense adhesions in 6 patients, iatrogenic enterotomies in 3 patients and difficult port insertion in 3 patients Table (3).

The mean operative time for LAP patients was 55 minutes (range 20-100). While the mean operative time for CONV patients was 91 minutes (range 55 -130). Patients who had a single band as the cause of the SBO had a mean operative time of 25 minutes (range 12-60). This was significantly less than the mean operative times for LAP and CONV procedures. Patients in the LAP group had a significantly shorter length of hospital stay (3 
days) compared to those patients who were converted to midline laparotomy (7 days).

Complications Table (4) included a total of 9 iatrogenic enterotomies 6 injuries during laparoscopy 3 of them were sutured laparoscopically and 3 cases were converted to open surgery. The other three enterotomies occurred during open adhesolysis and they were repaired. There were two wound infections in the CONV group. No wound infections or port-site hernias were identified in the LAP group.

Two patients (one in each group) didn't relieve postoperatively and were subjected to redo surgery through a midline laparotomy in the early postoperative period, Two patients in the CONV group had readmission to the hospital for recurrent partial SBO, but resolved with conservative measures. None of the patients who had intestinal injuries developed intestinal leakage. There was no operative mortality (within 30 days) in either group.

\section{Discussion:}

Laparoscopic adhesiolysis has been demonstrated to be technically feasible in small bowel obstruction and carries advantages in terms of post-surgical course. 7

In this study, Successful laparoscopic approach was obtained in $73 \%$ of cases and converted to open surgery in $27 \%$ of cases. These results are close to results recorded by many literatures. $3,5.8,9,10,11,12$

We found isolated band in 38 patients $(67.8 \%)$ most of them 33 patients were treated laparoscopically with short operative times. Only five patients of them $(13 \%)$ required conversion and this agrees with the results recorded by many authors. $3,6,9,11,13,14,15$

In our study, the predictive factors for successful laparoscopic adhesiolysis were single previous laparotomy, appendectomy as previous surgical operation, non-median previous laparotomy and early laparoscopic management within 48 hours from the onset of symptoms. Marc et a ${ }^{16}$ conducted a study similar to our study and concluded that, it is of outmost importance to focus on the number of previous surgery before proceedings to laparoscopic approach and patients with one or two previous operations are candidate for laparoscopic adhesiolysis.

In a study done by Cirocchi $\mathrm{R}$ et $\mathrm{a}^{\mathrm{l} 2}$ revealed no difference between open and laparoscopic groups in respect to iatrogenic injuries to bowel. In our series, iatrogenic perforations occurred in both open and laparoscopic procedures we recorded nine cases of iatrogenic small bowel injuries 6 cases (15\%) during laparoscopy and 3 cases $(18 \%)$ during open adhesiolysis. In general, iatrogenic injuries were more likely with dense and extensive adhesions. Although we tried to avoid perforations by using atraumatic graspers, pushing instead of grasping, and avoiding direct grasping of serosa, however they did occur.

In this study, there were two cases of early recurrence of adhesive obstruction only in the open (CONV) group this matches with the results of many authors $2,3,9,13$ who also demonstrated that the adhesions form after laparoscopic procedures much less than after conventional laparotomy.

In our study, we excluded cases that were suspected or proved to be due to malignant cause. This is because that obstruction would not be primarily due to adhesions only, but, rather, from tumor invasion or bulk obstruction, and these patients are likely to need small bowel resections or bypass procedures which are complex procedures to be performed laparoscopically in acute settings. In contrary to this, Idelevich et al. ${ }^{17}$ reported $63 \%$ of their patients with malignancy had adhesions as the cause of obstruction two of them had a single band and did not require conversion to open, and he agrees with the policy of laparoscopy in all patients with acute small bowel obstruction as an initial step.

\section{Conclusion:}

Initial laparoscopy is both safe and effective in management of most cases of adhesive small bowel obstruction All patients determined to have clinical picture of adhesive small bowel obstruction should have a trial of laparoscopic adhesiolysis initially. If laparoscopic adhesolysis was successful, 


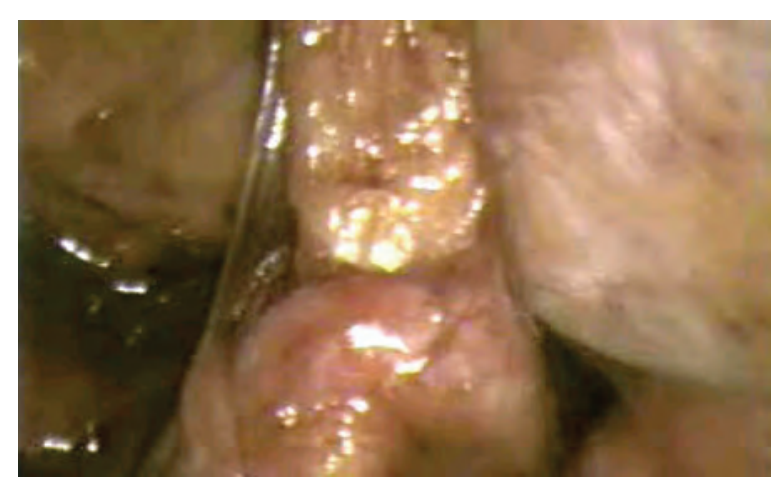

Figure (1)

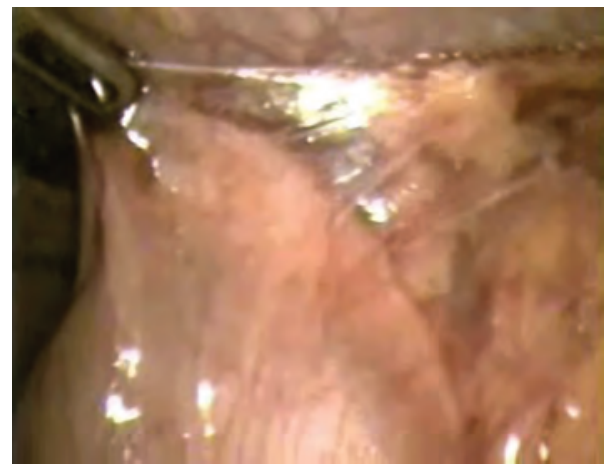

Figure (3)

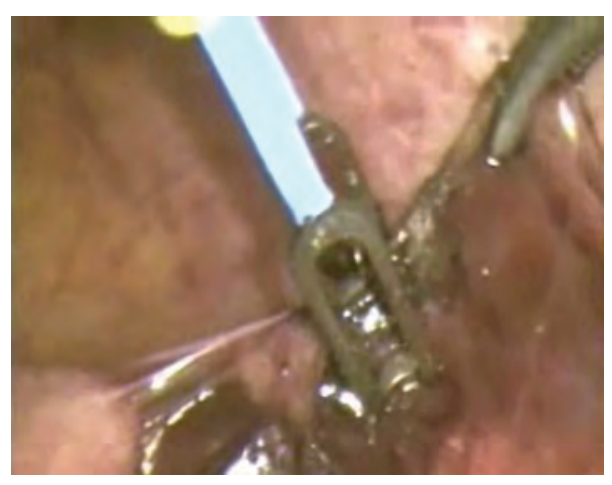

Figure (5)

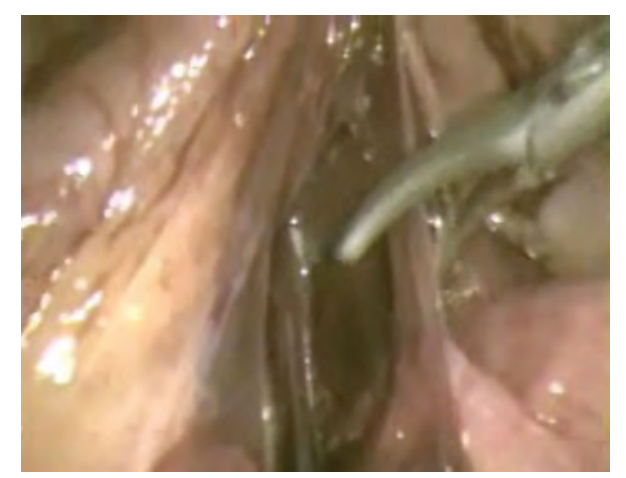

Figure (2)

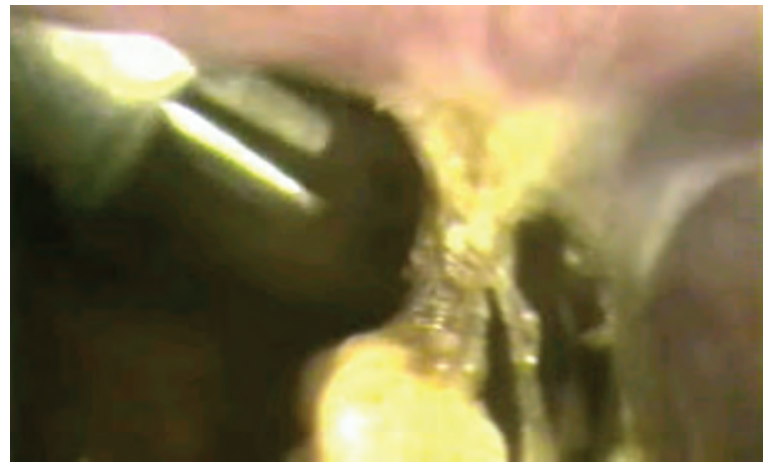

Figure (4)

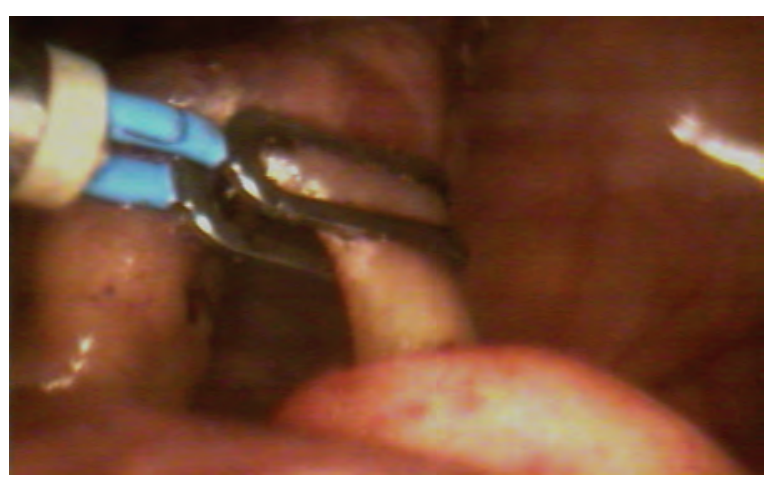

Figure (6)

Table 1: Shows the first former surgery in the two groups.

\begin{tabular}{|l|l|l|l|}
\hline The first former surgery & LAP group $(\mathbf{n}=\mathbf{4 0})$ & CONV group $(\mathbf{n}=\mathbf{1 6})$ & Total No of patients \\
\hline Appendicectomy & 29 & 8 & $37(66 \%)$ \\
\hline Hemicolectomy & 3 & 3 & $6(10.8 \%)$ \\
\hline Hysterectomy & 5 & 1 & $6(10.8 \%)$ \\
\hline Splenectomy & 1 & 2 & $3(5.3 \%)$ \\
\hline Umbilical hernia & 1 & 2 & $3(5.3 \%)$ \\
\hline Inguinal hernia & 1 & 0 & $1(1.8 \%)$ \\
\hline
\end{tabular}

patient would be spared a large laparotomy incisions and would benefit from shorter operative time, less adhesion formation, fewer wound complications (infection \& hernia), quicker recovery, shorter hospital stay, and less pain. 
Table (2): Shows the predictive factors for success of laparoscopic adhesiolysis.

\begin{tabular}{|l|l|l|}
\hline The predictive factors & LAP group (n= 40) & CONV group (n= 16) \\
\hline Number of previous laparotomy & 34 & 5 \\
Single laparotomy & 6 & 11 \\
More than one laparotomy & & \\
\hline Timing of surgery & 33 & 8 \\
Within 48 hrs & 7 & 8 \\
After 48 hrs & & \\
\hline Type of former operation & 29 & 8 \\
Appendicectomy & 3 & 3 \\
Hemicolectomy & 5 & 1 \\
Hysterectomy & 1 & 2 \\
Splenectomy & 1 & 2 \\
Umbilical hernia & 1 & 0 \\
Inguinal hernia & 13 & 9 \\
\hline Type of abdominal incision & 27 & 7 \\
Midline laparotomy & \multicolumn{2}{|l|}{} \\
Non- Midline laparotomy & 27 & \\
\hline
\end{tabular}

Table (3): Shows the causes of conversion to open surgery.

\begin{tabular}{|l|l|l|}
\hline Cause of conversion & Number of patients & Percentage \\
\hline Multiple serosal tears & 7 & $43 \%$ \\
\hline Dense adhesions & 3 & $19 \%$ \\
\hline Iatrogenic enterotomies & 3 & $19 \%$ \\
\hline Difficult cannulation & 3 & $19 \%$ \\
\hline
\end{tabular}

Table (4): Shows the incidence of complications in each group.

\begin{tabular}{|l|l|l|l|}
\hline \multicolumn{1}{|c|}{ The complications } & \multicolumn{1}{|c|}{$\begin{array}{c}\text { LAP group } \\
(\mathbf{n = 4 0 )}\end{array}$} & \multicolumn{1}{|c|}{$\begin{array}{c}\text { CONV group } \\
(\mathbf{n = 1 6 )}\end{array}$} & $\begin{array}{c}\text { Total No of } \\
\text { patients }\end{array}$ \\
\hline Iatrogenic intestinal injuries & $6(15 \%)$ & $3(18 \%)$ & $9(16 \%)$ \\
\hline No relief \& redo surgery & $1(2.5 \%)$ & $1(6.25 \%)$ & $2(3.6 \%)$ \\
\hline Wound infection & $0(0 \%)$ & $2(12.5 \%)$ & $2(3.6 \%)$ \\
\hline Early adhesive obstruction & $0(0 \%)$ & $2(12.5 \%)$ & $2(3.6 \%)$ \\
\hline
\end{tabular}

\section{Recommendation}

Long-term follow-up of patients is recommended to evaluate the impact of laparoscopy on the recurrence of adhesions and small bowel obstruction.

\section{Reference:}

1- Catena F, Di Saverio S, Kelly MD, Biffl WL, Ansaloni L, Mandalà V, et al: Bologna Guidelines for diagnosis and management of adhesive small bowel obstruction (ASBO): 2010 Evidence-Based Guidelines of the
World Society of Emergency Surgery. World J Emerg Surg 2011; 6: 5-12.

2- Cirocchi R, Abraha I, Farinella E, Montedori A, Sciannameo F: Laparoscopic versus open surgery in small bowel obstruction. Cochrane Database Syst Rev 2010; 17(2): CD007511. Review.

3- Li MZ, Lian L, Xiao LB, Wu WH, He YL, Song XM: Laparoscopic versus open adhesiolysis in patients with adhesive small intestinal obstruction. A systemic review and meta-analysis. American Journal of Surgery 2012; 204(5): 779-786. 
4- Zielinski MD, Eiken PW, Bannon MP, Heller SF, Lohse CM, Huebner M, Sarr MG: Small bowel obstruction-who needs an operation? A multivariate prediction model. World $J$ Surg 2010; 34(5): 910-919.

5- Nagle A, Ujiki M, Denham W, Murayama K: Laparoscopic adhesiolysis for small bowel obstruction. Am J Surg 2004; 187: 464-474.

6- Bailey IS, Rhodes M, O'Rourke N, Nathanson L, Fielding G: Laparoscopic management of acute small bowel obstruction. Br J Surg 1998; 85: 84-87.

7- Catena F, Resin D, Kelly MD, Biffl WL: Bologna guidelines for diagnosis and management of adhesive small bowel obstruction (ASBO): 2010 EvidenceBased Guidelines of the World Society of Emergency Surgery. World J Emerg Surg 2011; 6: 5-11.

8- Lujan HJ, Oren A, Plasencia G, Canelon G, Gomez E, Hernandez-Cano A, Jacobs M: Laparoscopic management as the initial treatment of acute small bowel obstruction. JSLS 2006; 10(4): 466-472.

9- Khaikin M, Schneidereit N, Cera S, Sands D, Efron J, Weiss EG, Nogueras JJ, Vernava AMIII, Wexner SD: Laparoscopic vs. Open surgery for acute adhesive smallbowel obstruction: Patients' outcome and cost-effectiveness. Surg Endosc 2007; 21: 742-753.
10- Rosin D, Kuriansky J, Bar Zakai B, Shabtai M, Ayalon A: Laparoscopic approach to small-bowel obstruction. J Laparoendosc Adv Surg Tech 2000; 5: 253-254.

11- Cavaliere D, Schirru A, Caristo I, Bianchi M, Cosce U, Cavaliere P: Laparoscopic management of small-bowel obstruction. Chir Ital 2005; 57: 215-225.

12- Wullstein C, Gross E: Laparoscopic compared with conventional treatment of acute adhesive small bowel obstruction. $\mathrm{Br} J$ Surg 2003; 90: 1147-1156.

13- Farinella E, Cirocchi R, La Mura F, Morelli U, Cattorin L, Delmonaco P, Migliaccio C, De Sol AA, Cozzaglio L, Sciannameo F: Feasibility of laparoscopy in small bowel obstruction. Research article. World J Emerg Surg 2009; 4: 3-17.

14- Ghosheh B, Salameh JR: Laparoscopic approach to acute small bowel obstruction: Review of 1061 cases. Surg Endosc 2007; 21: 45-58.

15- Tsumara H: Laparoscopic treatment of small bowel obstruction. Adhesion 2006; 9: 17-19.

16- Neff M, Schmidt B: Laparoscopic Treatment of a Postoperative Small Bowel Obstruction. Journal of society of laparoendoscopic surgeons (JSLS) 2010; 14(1): 133-136.

17- Idelevich E, Kashtan H, Mavor E, Brenner B: Small bowel obstruction caused by secondary tumors. Surg Oncol 2006; 15: 29-32. 